\title{
Fuzzy Optimum Design of Engine Room of Ship with Dynamic Response Using Genetic Algorithms
}

\author{
by Mitsuru Kitamura*, Member Hisashi Nobukawa*, Member \\ Fengxiang Yang**, Student Member
}

\begin{abstract}
In this paper, the theory of fuzzy set is introduced to the optimization of engine room structure considering the dynamic response constraints. Two ways of solving the fuzzy optimization are investigated. Firstly, the constraints of static stresses and dynamic response to the external excitation are handled by the fuzzy set theory. By this approach, a family of optimal designs under different degrees of satisfaction with constraints for the structure can be obtained. Secondly, the objective is also considered to be a fuzzy variable with its own membership function. Through maximizing the degree of membership to the fuzzy decision, a crisp solution can be provided to designers. A Genetic Algorithm (GA) is applied to do the optimization. The optimum results for the numerical example are presented and it is shown that the proposed method for the dynamic response optimization is applicable.
\end{abstract}

\section{Introduction}

Reference (10) provided the optimization of engine room structure considering the constraints on the stresses and dynamic response. The goal of the constraints is to keep the static and dynamic response of the structure not to be larger than the exact allowable values. However, in practice, it is not appropriate to deny a design absolutely just because the stresses of few members or the accelerations of few nodal points are slightly larger than their allowable values. For example, as to the allowable stress of $1800 \mathrm{kgf} / \mathrm{cm}^{2}$, all the designs whose maximal stresses are smaller than or equal to it are allowable, but if the maximal stress of the structure reaches $1801 \mathrm{kgf} / \mathrm{cm}^{2}$, according to the traditional method, this candidate design is unacceptable. However, there is no substantial difference between 1800 $\mathrm{kgf} / \mathrm{cm}^{2}$ and $1801 \mathrm{kgf} / \mathrm{cm}^{2}$ in practice. As a matter of fact, because a lot of uncertainties exist in ship structural design, many constraints are fuzzy in a certain sense. There are no well-defined boundaries between safety and unsafety. It is more reasonable that there should be transition stages from absolute safety to absolute unsafety.

Fuzzy set theory ${ }^{1,2)}$ appeared in 1965 and has been applied to a wide variety of scientific areas recently.

* Faculty of Engineering, Hiroshima University

** Graduate School, Hiroshima University

Received 8th July 1999

Read at the Autumn meeting 18, 19th Nov. 1999
However, real applications ${ }^{3), 4)}$ of it to structural optimization design are still relatively rare. This theory enables one to handle the fuzziness existing in engineering. In this paper, the constraints on stresses, displacements and accelerations of the structure, and the objective are considered to be fuzzy. Using the fuzzy set concept, the allowable stress is not fixed at an exact value. The membership function is introduced to describe the fuzziness of constraints. According to the membership function, the degree of membership function for a constraint can be known and can be understood as the degree of its satisfaction. For the example mentioned above, the degree of satisfaction with the stress constraints in which the maximal stress reaches $1800 \mathrm{kgf} / \mathrm{cm}^{2}$ can be 1.0 , while the one of $1801 \mathrm{kgf} / \mathrm{cm}^{2}$ may be considered to be 0.9 . Even though the degree of satisfaction 0.9 with the stress constraints is smaller than 1.0, the design is still in the allowable domain. If the maximal stress reaches the absolutely unacceptable value, the membership degree of this fuzzy constraints should be defined to be 0 .

There are two models in solving the structural optimization problems in fuzzy environment. One is to compute the optimal values of the objective for all levels of satisfaction with the fuzzy constraints. Consequently a family of optimal design under different degrees of satisfaction with the constraints can be obtained. Therefore, designers can choose the optimal design among them basing on their experience. The other is to introduce the fuzzy set concept into the objective. Namely designers have the fuzzy goal for the problem. The objective has its own membership function. In this 
case, a crisp solution can be obtained in which the membership degree of fuzzy decision is maximized.

It is necessary to solve the ordinary optimization problems appearing in both of the two approaches. GAs are chosen to be the optimization method in this study. Based on the work of Reference(10), the same operators and the floating point representation to a string of solution are also applied to this calculation.

\section{Illustration of Problem}

The objective is to find the design variables $\boldsymbol{X}$ to minimize the cost of structure under the fuzzy constraints as the following :

$$
\tilde{f}(\boldsymbol{X}) \rightarrow \min
$$

subject to :

(1) Stress constraints

$$
\left|\sigma_{i, \max }^{s}\right|+\left|\sigma_{i, \max }^{d}\right| \tilde{\subset} \widetilde{C}_{\sigma}^{i} \quad i=1,2, \cdots, n e
$$

where the $\sigma_{i, \max }^{s}$ and $\sigma_{i, \max }^{d}$ are the static and dynamic maximal stress of element $i$, respectively. $\widetilde{C}_{\delta}^{j}$ is the allowable fuzzy domain of stress for element $i$.

(2) Displacement constraints

$$
\left|Y_{j}\right|+\left|U_{j}\right| \widetilde{\subset} \widetilde{C}_{D}^{j} \quad j=1,2, \cdots, n j
$$

where $Y_{j}$ and $U_{j}$ are the maximal dynamic and static displacement of node $j$, respectively. $\widetilde{C}_{D}^{j}$ is the allowable fuzzy domain of displacement for node $j$.

(3) Acceleration constraints

$$
\left|\bar{a}_{j}\right| \tilde{\subset} \widetilde{C}_{a}^{j} \quad j=1,2, \cdots, n j
$$

where $\bar{a}_{j}$ and $\widetilde{C}_{a}^{j}$ are the maximal acceleration and its allowable fuzzy domain of node $j$, respectively.

(4) Design variable constraints

$$
x_{i, \min } \leq x_{i} \leq x_{i, \max } \quad i=1,2, \cdots, q
$$

The fuzzy constraints (2), (3) and (4) can be generalized as

$$
g_{i}(\boldsymbol{X}) \tilde{\subset} \widetilde{C}_{g}^{j} \quad i=1,2, \cdots, m
$$

It is noticed that there are some differences between the conventional optimization and the fuzzy one in expression. Formula (1) represents the fuzzy goal of the problem such that $f(\boldsymbol{X})$ should be minimized in a fuzzy sense. The symbol $\leq$ in conventional optimization has been replaced by $\tilde{\subset}$ in fuzzy optimization. $\tilde{\subset}$ in formula (6) means that $g_{j}(\boldsymbol{X})$ is a member of the fuzzy subset $\widetilde{C}_{g}^{j}$ in the sense of $\mu_{C}\left(g_{j}(\boldsymbol{X})\right)>0$, in which $\mu_{C}\left(g_{j}(\boldsymbol{X})\right)$ is the membership degree of constraint $g_{j}(\boldsymbol{X})$. The exact allowable value is replaced by $\widetilde{C}_{g}^{j}$ which is the allowable fuzzy domain of $g_{j}(\boldsymbol{X})$.

The maximum static stress in each element and the static displacement of every node can be calculated by solving the static equilibrium equation as below.

$$
\boldsymbol{K} \boldsymbol{Y}=\boldsymbol{P}
$$

The maximum dynamic stress in each element, and the dynamic displacement and acceleration of nodal points can be calculated through the dynamic analysis described in the next chapter.

\section{Dynamic Analysis}

The differential equations of motion for the engine room structure under the excitation $\boldsymbol{F}(t)$ can be written as below:

$$
\boldsymbol{M} \ddot{\boldsymbol{U}}+\boldsymbol{C} \dot{\boldsymbol{U}}+\boldsymbol{K} \boldsymbol{U}=\boldsymbol{F}(t)
$$

where $\boldsymbol{M}, \boldsymbol{C}, \boldsymbol{K}$ are the mass, damping and total stiffness matrix, respectively. The dimensions of these matrices are $n \times n$, where $n$ is the number of degrees of freedom of the structure. The damping is assumed to be proportional to the stiffness and mass of structure. Based on these simplifications, the equations can be solved by modal superposition principle as the following :

Suppose that the first $p$ low-order modes of the structure, which can be represented by mode-shape matrix $\boldsymbol{\Phi}$, are obtained by solving the frequency problem, with introducing the coordinate transformation

$$
\boldsymbol{U}=\boldsymbol{\Phi}_{\boldsymbol{q}}
$$

Eq. 8 can be transformed into $p$ uncoupled differential equations as

$$
\boldsymbol{M}^{*} \ddot{\boldsymbol{q}}+\boldsymbol{C}^{*} \dot{\boldsymbol{q}}+\boldsymbol{K}^{*} \boldsymbol{q}=\boldsymbol{F}^{*}(t)
$$

where

$$
\begin{aligned}
& \boldsymbol{M}^{*}=\boldsymbol{\Phi}^{T} \boldsymbol{M} \boldsymbol{\Phi}=\boldsymbol{I} \\
& \boldsymbol{C}^{*}=\boldsymbol{\Phi}^{T} \boldsymbol{C} \boldsymbol{\Phi}=\operatorname{diag}\left[2 \xi_{i} \omega_{i}\right] \\
& \boldsymbol{K}^{*}=\boldsymbol{\Phi}^{T} \boldsymbol{K} \boldsymbol{\Phi}=\operatorname{diag}\left[\omega_{i}^{2}\right] \\
& \boldsymbol{F}^{*}(t)=\boldsymbol{\Phi}^{T} \boldsymbol{F}(t)
\end{aligned}
$$

For every uncoupled equation, the solution can be given in the form of the convolution integral generally. In this study, since the vibration of engine room structure is caused mainly by the propeller, the excitation is simplified as

$$
\boldsymbol{F}(t)=\overline{\boldsymbol{F}} \times \sin (\bar{\omega} t+\beta)
$$

where $\overline{\boldsymbol{F}}$ and $\bar{\omega}$ are the amplitude and frequency of excitation, respectively.

With this form of excitation, when the vibration reaches the stable state, all the stresses of elements, displacements and accelerations vibrate with frequency $\bar{\omega}$. The maximal values of them can be calculated by the mode superposition principle without time $t$. These maximum values are independent of the time $t$. As a result, $t$ does not appear in the optimization process.

Eq. 10 can be written separately as below :

$$
\begin{aligned}
& \ddot{q}_{i}+2 \xi_{i} \omega_{i} \dot{q}_{i}+\omega_{i}^{2} q_{i}=\bar{F}_{i}^{*} \sin (\bar{\omega} t+\beta) \\
& i=1,2, \cdots, p
\end{aligned}
$$

Suppose $\gamma_{i}=\bar{\omega} / \omega_{i}$, the amplitude of normal coordinate $q_{i}$ can be obtained as

$$
A_{i}=\frac{\bar{F}_{i}^{*}}{\omega_{i}^{2} \sqrt{\left(1-\gamma_{i}^{2}\right)^{2}+\left(2 \xi_{i} \gamma_{i}\right)^{2}}}
$$

When the normal coordinates are obtained, the origi-. nal displacements of nodes can be calculated through Eq. (9). Accordingly, the dynamic stresses of elements are computed according to the displacements as in the static analysis. In vibration control problems, the accelerations of certain points in the structure should be included in the constraints. The accelerations of nodes can be derived from the displacements. Their amplitudes are as below:

$$
|\dot{\boldsymbol{U}}|_{\max }=\bar{\omega}^{2} \boldsymbol{\Phi} \boldsymbol{A}
$$

where

$$
\boldsymbol{A}=\left\{A_{1}, A_{2}, \cdots, A_{p}\right\}^{T}
$$


In this study, damping is taken as the following form :

$$
\boldsymbol{C}=\eta_{1} \boldsymbol{M}+\eta_{2} \boldsymbol{K}
$$

therefore,

$$
C^{*}=\operatorname{diag}\left[\eta_{1}+\eta_{2} \omega_{i}^{2}\right]
$$

From this equation

$$
\xi_{i}=\frac{\eta_{1}}{2 \omega_{i}}+\frac{\eta_{2} \omega_{i}}{2}
$$

For ship vibration problems, $\eta_{1}$ and $\eta_{2}$ can be taken 0 . 034 and 0.001 , respectively ${ }^{13}$.

\section{Fuzzy Optimization}

\section{1 Fuzzy Constraints}

The value of the membership function of constraints should be 0 if the constraints are strongly violated, and 1 if they are well satisfied. The membership function should decrease monotonously from 1 to 0 . It can be taken the linear form or non-linear form. Here the linear form is introduced as Fig. 1. Its formula is given as below :

$$
\mu_{C}\left(g_{j}\right)= \begin{cases}1 & g_{j}<g^{l} \\ 1-\frac{g_{j}-g_{j}^{l}}{g_{j}^{u}-g_{j}^{l}} & g_{j}^{l} \leq g_{j} \leq g_{j}^{u} \\ 0 & g_{j}>g_{j}^{u}\end{cases}
$$

where $g_{j}^{u}$ and $g_{j}^{l}$ represent the values of $g_{j}(\boldsymbol{X})$ such that the degree of the membership function $\mu_{c}\left(g_{j}\right)$ is 0 and 1 , respectively within the fuzzy domain as shown in Fig. 1. In this study, the stress, displacement and acceleration constraints are also handled in this way. It can be understood from Fig. 1 that the membership degree of the constraints represents the degree of the satisfaction with the constraints. From this sense, this value looks like a safety factor. Here smaller violations of the strict constraints in the conventional methods are acceptable and are illustrated by this degree of satisfaction. Obviously the larger this degree is, the safer the design is. For the design variable constraints, the crisp form is acceptable. This problem has mixed constraints - the fuzzy and the crisp.

\section{2 Fuzzy Solution}

For the fuzzy optimization problem expressed by formulation ( 1 ) and ( 6 ), the fuzzy feasible region is the intersection of all the constraints

$$
\widetilde{S}=\bigcap_{j=1}^{m} C_{g}^{j}
$$

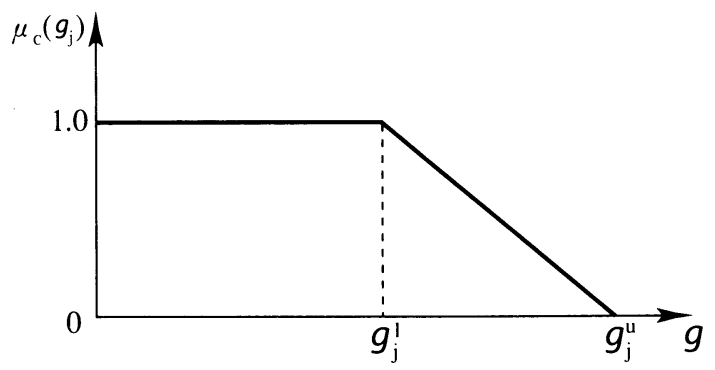

Fig. 1 Membership Function of Constraint
The membership degree of any candidate design corresponding to the variables $\boldsymbol{X}$ to $\widetilde{S}$ is the minimum degree of satisfaction with all of the constraints, written as

$$
\mu_{S}(\boldsymbol{X})=\min _{j=1, \cdots, m}\left\{\mu_{C}\left(g_{j}(\boldsymbol{X})\right)\right\}
$$

According to the decomposition theorem, the fuzzy constraints set can be decomposed into a series of $\alpha^{-}$ level ordinary sets. In other words, it can be viewed as a family of ordinary sets. Considering the $\alpha$-level set of the fuzzy constraints $\widetilde{C}_{g}^{j}$,

$$
C_{\alpha}=\left\{g_{j} \mid g_{j} \in R^{n}, \mu_{C}\left(g_{j}\right) \geq \alpha\right\}, \forall \alpha \in[0,1]
$$

The $\alpha$-level set is an ordinary set whose elements belong to the corresponding fuzzy set to a certain degree $\alpha$. $\alpha$ can be called the fortified value for the design. Under this degree of safety, the fuzzy optimization problem can be transformed into an unfuzzy one as below :

$$
\min f(\boldsymbol{X})
$$

subject to :

$$
\begin{aligned}
& \alpha \leq \mu_{C}\left(g_{j}\right)=1-\frac{g_{j}-g^{l}}{g^{u}-g^{l}} \\
& j=1,2, \cdots, m
\end{aligned}
$$

At different $\alpha \in[0,1]$, the objective $f(\boldsymbol{X})$ can be optimized by any efficient algorithms for classical optimization. According to this, the problem described by formulation (1) to (5) can be re-written as the following form :

Giving a value of $\alpha \in[0,1]$, $\min f(\boldsymbol{X})$

subject to :

(1) Stress constraints

$$
\begin{aligned}
& \left|\sigma_{i, \max }^{S}\right|+\left|\sigma_{i, \max }^{d}\right| \leq \sigma^{u}-\alpha\left(\sigma^{u}-\sigma^{l}\right) \\
& i=1,2, \cdots, \text { ne }
\end{aligned}
$$

(2) Displacement constraints

$$
\begin{aligned}
& \left|Y_{j}\right|+\left|U_{j}\right| \leq D^{u}-\alpha\left(D^{u}-D^{l}\right) \\
& j=1,2, \cdots, n j
\end{aligned}
$$

(3) Acceleration constraints

$$
\left|\bar{a}_{j}\right| \leq a^{u}-\alpha\left(a^{u}-a^{l}\right) \quad j=1,2, \cdots, n j
$$

(4) Design variable constraints

$$
x_{i, \min } \leq x_{i} \leq x_{i, \max } \quad i=1,2, \cdots, q
$$

where $\sigma^{u}, D^{u}, a^{u}$ are the upper limits of the allowable stress, displacement and acceleration, respectively. $\sigma^{\iota}$, $D^{l}, a^{l}$ are the lower limits of them illustrated in Fig. 1. Solving this parametric optimization problem, the curve representing the optimal designs with respect to different $\alpha$ can be drawn.

\section{3 Crisp Solution}

How to make use of the fuzzy solution described above is a problem. In fact, the fuzzy solution described above consists of an infinite number of points. At any tolerable fortified level, there is an optimal design. The lower the fortified level is, the smaller the objective of optimal structure is. This means the degree of satisfaction with constraints and the objective conflict with each other. In practice, it is expected to design a structure with highest fortified level, and, at the same time, smallest objective value. It is difficult for 
designers to decide which is the one they want among all the optimal designs under different fortified levels. As a matter of fact, the objective is not considered fuzzy in the approach above. At an exact fortified level, there are only two choices, either the optimal solution or the one which is not the optimal solution. In fact, all the solutions obtained by the method above are the optimal ones with different fortified levels. In practice, designers have to select a compromise solution from them based on their subjective judgment. This means designers just want to achieve their goal in the fuzzy sense. It is natural to assume that designers have imprecise or fuzzy goal for the objective. Therefore, in this approach, the optimized objective is also quantified by eliciting a corresponding membership function. In order to define the membership function, the results of the fuzzy solution in the last section can be used. For example, as shown in Fig. 2, the value of membership function of objective obtained at the fortified level of constraints $\alpha=0$ in the above fuzzy solution can be defined to be 1 , which is the smallest objective in the calculation. The one at $\alpha=1$ to be 0 . The linear form of the membership function of the objective is introduced in this study as

$$
\mu_{F}(f)= \begin{cases}1 & f<f^{\iota} \\ 1-\frac{f-f^{l}}{f^{u}-f^{l}} & f^{\iota} \leq f \leq f^{u} \\ 0 & f>f^{u}\end{cases}
$$

The fuzzy goal $\widetilde{F}$ and the fuzzy constraints $\widetilde{C}$ combine to form the fuzzy decision $\widetilde{D}$ which is a fuzzy set resulting from the intersection of $\widetilde{F}$ and $\widetilde{C}$. Namely

$$
\widetilde{D}=\widetilde{F} \cap \widetilde{C}
$$

The membership function of fuzzy decision can be defined by

$$
\mu_{D}=\min \left\{\mu_{F}, \mu_{C}\right\}
$$

In this sense, the crisp optimal solution can be obtained by

maximizing $\mu_{D}(\boldsymbol{X}), \boldsymbol{X} \in D$.

The value of $\mu_{D}(\boldsymbol{X})$ can be interpreted as representing an overall degree of satisfaction with the fuzzy goal and fuzzy constraints. By introducing the auxiliary variable $\lambda$ which corresponds to $\mu_{D}(\boldsymbol{X})$, the problem can be transformed into the following equivalent conventional nonlinear programming problem as:

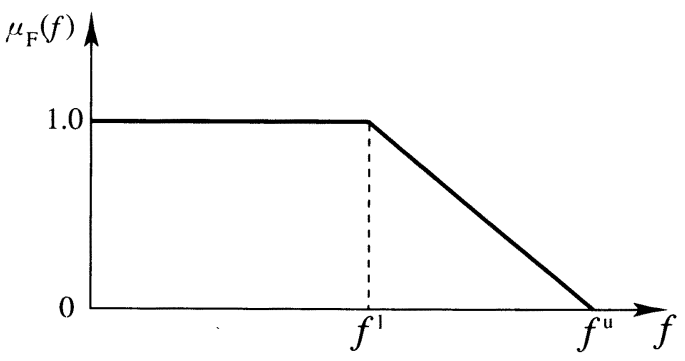

Fig. 2 Membership Function of Objective $\operatorname{maximize} \lambda$

subject to the constraints explained below :

(1) When the value of membership function of objective is $\lambda$, according to Eq. (32), the objective is

$$
\bar{f}=f^{u}-\lambda\left(f^{u}-f^{\imath}\right)
$$

It is desirable to find the design whose objective is smaller than or equal to $\bar{f}$, which can be expressed as

$$
f \leq f^{u}-\lambda\left(f^{u}-f^{l}\right)
$$

This inequality is the constraint on the objective. $f^{l}$ and $f^{u}$ are the lower and upper bounds of objective, respectively. They can be determined by designers or by the calculation of the fuzzy solution described in the last section. Of course the lower bound of objective for minimum problems should be determined reasonably.

(2) The constraints on the static and dynamic response are following.

(a) Stress constraints

$$
\begin{aligned}
& \left|\sigma_{i, \max }^{S}\right|+\left|\sigma_{i, \max }^{d}\right| \leq \sigma^{u}-\lambda\left(\sigma^{u}-\sigma^{l}\right) \\
& i=1,2, \cdots, \text { ne }
\end{aligned}
$$

(b) Displacement constraints

$$
\begin{aligned}
& \left|Y_{j}\right|+\left|U_{j}\right| \leq D^{u}-\lambda\left(D^{u}-D^{\prime}\right) \\
& j=1,2, \cdots, n j
\end{aligned}
$$

(c) Acceleration constraints

$$
\left|\bar{a}_{j}\right| \leq a^{u}-\lambda\left(a^{u}-a^{l}\right) \quad j=1,2, \cdots, n j
$$

(d) Design variable constraints

$$
x_{i, \min } \leq x_{i} \leq x_{i, \max } \quad i=1,2, \cdots, q
$$

where $\sigma^{u}, D^{u}, a^{u}, \sigma^{l}, D^{l}, a^{l}$ are the same as that of the last section.

It is significant to realize from the crisp solution that both the fuzzy goal and the fuzzy constraints are included into the constraints in exactly the same way. Therefore, for fuzzy decision, there is no difference between the fuzzy goal and fuzzy constraints. The fuzzy goal becomes a constraint. This indicates that it is possible to deal with the multiobjective optimization problems with this method.

\section{Genetic Algorithms}

GAs are the powerful tools to optimize functions. They can be applied to the structural optimal design with complex constraints. Reference (10) investigated the applicability of $\mathrm{GAs}^{5,6), 7)}$ with floating point representation of string to the engine room optimization problem under the constraints of static and dynamic response. Some operators were introduced in that study. In this study, the same GA with the same operators is also used to solve the ordinary optimization problems appearing in the two approaches described above. The operators include simple crossover, arith metical crossover, uniform mutation, boundary mutation, and non-uniform mutation.

\section{1 Application in Fuzzy Solution}

For the fuzzy optimization problems obtained in the above two approaches, it can be known that they involve a large number of constraints. In the approach of the fuzzy solution, constraint handling can be done by penalty methods ${ }^{8), 99,10)}$ which use penalty functions as 
an adjustment to the optimized objective function. Therefore, a constrained problem is transformed to an unconstrained problem by associating a penalty with all constraint violations. Thus the formula (27) above is transformed into the optimization of function :

$$
\min F(\boldsymbol{X}, \alpha)=f(\boldsymbol{X})+\sum_{i=1}^{m} \delta_{i} \phi_{i}(\boldsymbol{X}, \alpha)
$$

where $m$ is the total number of constraints, $\delta_{i}$ is the penalty coefficient of constraint $i, \phi_{i}(\boldsymbol{X}, \alpha)$ is a penalty term related to the $i$-th constraint $(i=1, \cdots, m)$.

The minimum problem like formula (41) can be transformed to be the maximum one by adding the negative sign. In GAs, the fitness should be positive, therefore, formula (41) is transformed to the maximum problem by adding the negative sign and a constant as below :

$$
\bar{F}(\boldsymbol{X}, \alpha)= \begin{cases}\varepsilon & C \leq F(\boldsymbol{X}, \alpha) \\ C-F(\boldsymbol{X}, \alpha) & C>F(\boldsymbol{X}, \alpha)\end{cases}
$$

where $C$ is a positive constant generally larger than $F(\boldsymbol{X}, \alpha)$ and $\varepsilon$ is a small positive value. The detailed procedure can be found in Reference (10).

The penalty terms for formula (41) can be calculated as the following :

(1) Penalty term of stress constraints

$$
\phi_{S}^{\alpha}=\sum_{i=1}^{n e}\left(\max \left\{0, \frac{\left|\sigma_{i, \max }^{S}\right|+\left|\sigma_{i, \max }^{d}\right|}{\sigma^{u}-\alpha\left(\sigma^{u}-\sigma^{l}\right)}-1.0\right\}\right)
$$

(2) Penalty term of displacement constraints

$$
\phi_{d}^{\alpha}=\sum_{j=1}^{n_{j}}\left(\max \left\{0, \frac{\left|Y_{j}\right|+\left|U_{j}\right|}{D^{u}-\alpha\left(D^{u}-D^{l}\right)}-1.0\right\}\right)
$$

(3) Penalty term of acceleration constraints

$$
\phi_{a}^{\alpha}=\sum_{j=1}^{n j}\left(\max \left\{0, \frac{\left|\bar{a}_{j}\right|}{a^{u}-\alpha\left(a^{u}-a^{l}\right)}-1.0\right\}\right)
$$

The meaning of all the mathematical characters can be found in Chapter 2 .

\section{2 Application in Crisp Solution}

In the approach of crisp solution, as shown in formula (35), it is a maximum problem. Therefore, $\lambda$ can be taken as the fitness. For every candidate design, based on the design variables, the minimum degree of the membership to the constraints and objective can be calculated according to their membership function after the results of structural analysis are obtained. This minimum value is the fitness of the candidate design. Among all the candidate designs which are called chromosomes in GAs, the chromosomes compete each other and the ones with higher fitness values will survive. The solution can be reached with generations. The penalty method is not needed.

For every candidate design, firstly the minimum membership degrees of objective and constraints for a design $q$ are calculated as below :

(1) Membership degree of objective

$$
\lambda^{0}= \begin{cases}1 & f<f^{l} \\ 1-\frac{f-f^{l}}{f^{u}-f^{l}} & f^{l} \leq f \leq f^{u} \\ 0 & f>f^{u}\end{cases}
$$

(2) Minimum membership degree of stress constraints

$$
\lambda^{\sigma}=\min _{i=1, \cdots, n e} \lambda_{i}^{\sigma}
$$

where $\lambda_{i}^{\sigma}$ can be calculated by the following formula. For convenience, let

$$
\sigma_{i, \max }^{s d}=\left|\sigma_{i, \max }^{s}\right|+\left|\sigma_{i, \max }^{d}\right|
$$

then

$$
\lambda_{i}^{\sigma}= \begin{cases}1 & \sigma_{i, \text { max }}^{\text {sd }}<\sigma^{l} \\ \frac{\sigma^{u}-\sigma_{i, \text { max }}^{\text {sd }}}{\sigma^{u}-\sigma^{l}} & \sigma^{l} \leq \sigma_{i, \text { max }}^{s d} \leq \sigma^{u} \\ 0 & \sigma_{i, \text { max }}^{\text {sd }}>\sigma^{u}\end{cases}
$$

(3) Minimum membership degree of displacement constraints

$$
\lambda^{d}=\min _{j=1, \cdots, n j} \lambda_{j}^{d}
$$

where

$$
\lambda_{j}^{d}= \begin{cases}1 & \left|Y_{j}\right|+\left|U_{j}\right|<D^{l} \\ \frac{D^{u}-\left(\left|Y_{j}\right|+\left|U_{j}\right|\right)}{D^{u}-D^{l}} & D^{l} \leq\left|Y_{j}\right|+\left|U_{j}\right| \leq D^{u} \\ 0 & \left|Y_{j}\right|+\left|U_{j}\right|>D^{u}\end{cases}
$$

(4) Minimum membership degree of acceleration constraints

$$
\lambda^{a}=\min _{j=1, \cdots, n j} \lambda_{j}^{a}
$$

where

$$
\lambda_{j}^{a}= \begin{cases}1 & \left|\bar{a}_{j}\right|<a^{l} \\ \frac{a^{u}-\left|\bar{a}_{j}\right|}{a^{u}-a^{l}} & a^{l} \leq\left|\bar{a}_{j}\right| \leq a^{u} \\ 0 & \left|\bar{a}_{j}\right|>a^{u}\end{cases}
$$

then, the membership degree of the fuzzy decision for this design $q$ is calculated to be the fitness of the design.

$$
\lambda^{q}=\min \left\{\lambda^{o}, \lambda^{\sigma}, \lambda^{d}, \lambda^{\alpha}\right\}
$$

\section{Numerical Example}

\section{1 Computing Model}

The principal dimensions of the ship for numerical example are :

Length P. $\mathrm{P}=70 \mathrm{~m}$; Breadth (mld.) $=12 \mathrm{~m}$;

Depth (mld. $)=7.12 \mathrm{~m}$; Draught $=4.14 \mathrm{~m}$;

Weight of main engine $=31$ tonf ;

Weight of generator $=2$ tonf $\times 2$ sets ;

$S H P=1800$ PS ; Blade number $=4$;

Propeller $D_{p}=2.40 \mathrm{~m} ; \mathrm{N}=284 \mathrm{rpm}$.

The simplified three-dimensional finite element model of the engine room part is given by Fig. 3 . The virtual added water mass is included in the dynamic analysis. The numbers of elements and nodal points of this model are 156 and 98, respectively.

The propeller excitation is assumed to act on the rear of the ship in vertical direction shown in Fig. 3. In this study, only the surface force in vertical direction is estimated according to the following formula ${ }^{11,12)}$.

$$
F_{z s}=\frac{K_{s} \cdot S H P}{(Z-1)\left(C / D_{p}\right)^{3 / 2} \cdot N \cdot D_{p}}
$$

where $K_{s}=0.031, C / D_{p}=0.25$.

The revolution of the main engine is $284 \mathrm{rpm}$, and the propeller has four blades. Accordingly, the frequency of the excitation is $18.933 \mathrm{~Hz}$. The static load condition is given in Fig. 4. $0.3 \mathrm{~m}$ water head acts on the upper deck and $100 \mathrm{kgf} / \mathrm{m}^{2}$ of machine weights are on the second 


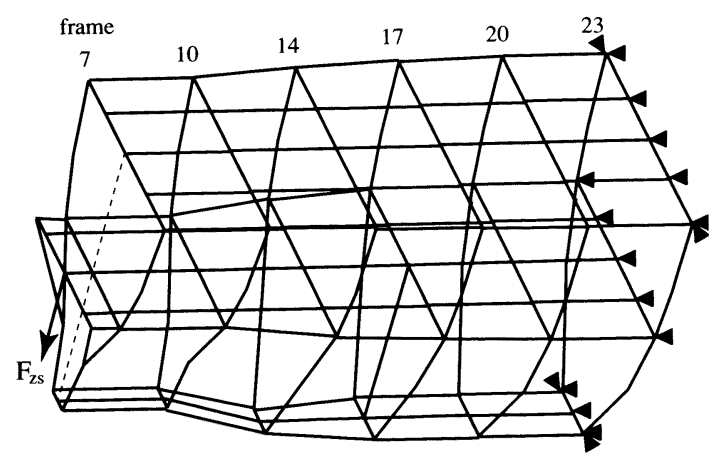

Fig. 3 Structural Model

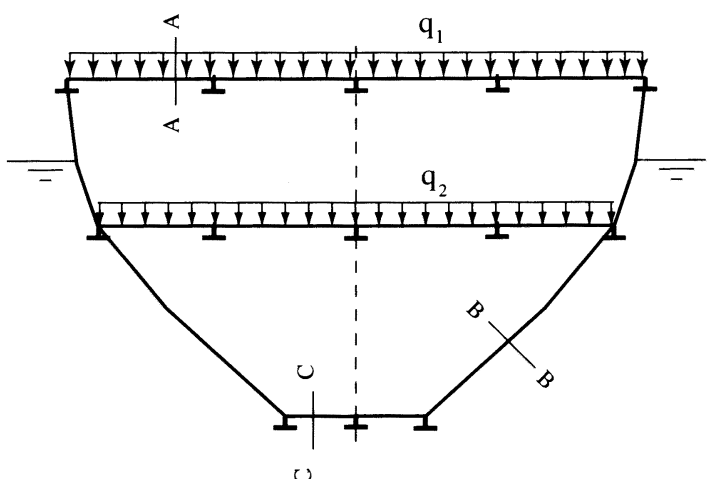

Static Load

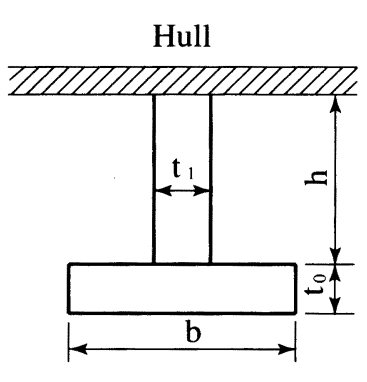

$\mathrm{A}-\mathrm{A}, \mathrm{B}-\mathrm{B}, \mathrm{C}-\mathrm{C}$

Fig. 4 Static Load and Design Variables

deck. All the original constraints are softened by admitting a certain violations. For the stress constraints, the allowable stress of $18 \mathrm{kgf} / \mathrm{mm}^{2}$ in conventional method is softened to be $18.00 \sim 18.10 \mathrm{kgf} / \mathrm{mm}^{2}$ here by introducing the membership function whose value is 0 when the stress reaches $18.10 \mathrm{kgf} / \mathrm{mm}^{2}$. The dynamic response constraints are also fuzzy and their tolerable acceleration is $37 \sim 41 \mathrm{gal}$ in this calculation.

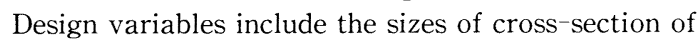
web beams and web frames in side and bottom of ship shown in Fig. 4. The cross-sections of all the web beams on the upper deck are taken the same. The side web frames and bottom ones are handled in the same
Table 1 Bound of Design Variables

\begin{tabular}{|c|c|c|c|c|}
\hline variable & $\mathrm{h}$ & $\mathrm{t}_{0}$ & $\mathrm{t}_{1}$ & $\mathrm{~b}$ \\
\hline $\begin{array}{c}\text { bound } \\
(\mathrm{mm})\end{array}$ & $200 \sim 600$ & $8 \sim 20$ & $8 \sim 16$ & $60 \sim 200$ \\
\hline
\end{tabular}

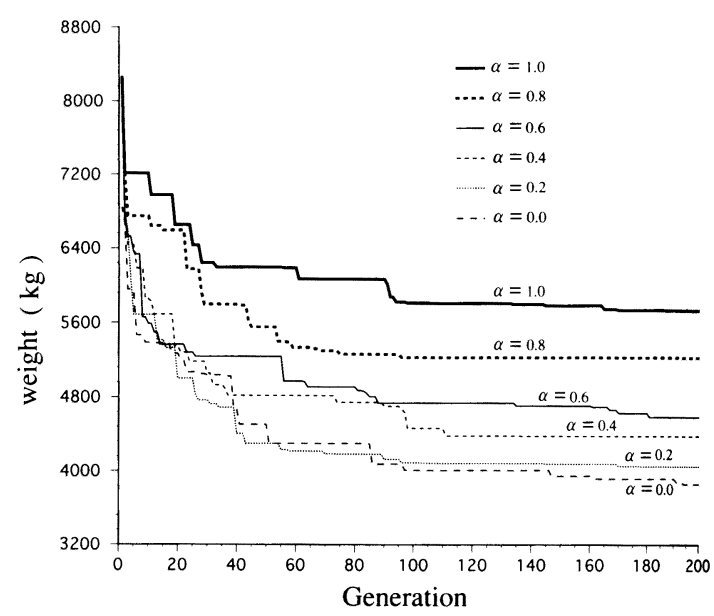

Fig. 5 History of GA at Different $\alpha$

Table 2 Results of Design Variables at Different $\alpha$

\begin{tabular}{|c|c|c|c|c|c|c|c|c|c|c|c|c|}
\hline & \multicolumn{3}{|c|}{ deck } & \multicolumn{1}{c|}{ side } & \multicolumn{4}{c|}{ bottom } \\
\hline$\alpha$ & $\mathrm{h}$ & $\mathrm{t}_{0}$ & $\mathrm{t}_{1}$ & $\mathrm{~b}$ & $\mathrm{~h}$ & $\mathrm{t}_{0}$ & $\mathrm{t}_{1}$ & $\mathrm{~b}$ & $\mathrm{~h}$ & $\mathrm{t}_{0}$ & $\mathrm{t}_{1}$ & $\mathrm{~b}$ \\
\hline 1.0 & 226.8 & 11.5 & 8.0 & 71.8 & 508.0 & 11.2 & 8.4 & 160.9 & 600.0 & 18.8 & 13.7 & 60.0 \\
\hline 0.8 & 226.8 & 13.3 & 8.3 & 61.1 & 433.6 & 11.8 & 8.0 & 182.1 & 528.4 & 11.0 & 8.0 & 179.1 \\
\hline 0.6 & 200.0 & 13.3 & 8.0 & 70.8 & 341.2 & 14.9 & 8.0 & 152.3 & 361.2 & 11.6 & 8.0 & 83.0 \\
\hline 0.4 & 200.0 & 10.4 & 8.0 & 89.4 & 331.6 & 8.00 & 9.3 & 200.0 & 287.2 & 13.3 & 9.4 & 78.2 \\
\hline 0.2 & 210.8 & 14.8 & 8.0 & 60.0 & 345.2 & 10.3 & 9.3 & 95.8 & 250.4 & 9.9 & 8.0 & 143.7 \\
\hline 0.0 & 203.1 & 14.3 & 8.0 & 65.0 & 400.0 & 11.2 & 8.0 & 60.0 & 287.2 & 11.0 & 8.0 & 112.2 \\
\hline
\end{tabular}

way. Therefore, there are only twelve design variables left. The bounds of them are listed in Table 1. For all the members, the bound of each size of cross-section is assumed the same.

\section{2 Optimization Results}

\subsubsection{Fuzzy Solution}

Giving $\alpha$ the different values, the optimal results can be obtained by using GAs. Here pop-size $=50$, probabil. ity of uniform mutation $p_{s m}$ is 0.1 , probability of nonuniform mutation $p_{n m}$ is 0.1 , coefficient $b=2$, probability of boundary mutation $p_{b m}$ is 0.05 , probability of simple crossover $p_{s c}$ is 0.50 , probability of arithmetic crossover $p_{a c}$ is 0.1 . Fig. 5 shows the history of the GA at different fortified levels.

The solutions at different $\alpha$ values are given in Table 2. From this Table, it is observed that most of the web thickness of stiffeners $\left(t_{1}\right)$ of the deck beams and side web frames reach their lower bound values at any $\alpha$ values. 
In Table $3, \quad a_{\alpha}^{0}$ and $\sigma_{\alpha}^{0}$ represent the allowable acceleration and stress at the fortified level $\alpha$. It can be seen that when $\alpha$ decreases, the corresponding allowable stress and acceleration increases. Therefore, the value of the objective of the optimal solution decreases with the decrease of $\alpha$. This relationship is shown in Fig. 6. From Fig. 6, it can be observed that with the increase of $\alpha$ from 0.4 to 0.6 , the objective value increases slowly. It is reasonable for designers to have a preference of the design with $\alpha=0.6$ over the one with $\alpha=0.4$ because the design with $\alpha=0.6$ is safer without sacrificing the objective too much.

\subsection{Crisp Solution}

All the results obtained in fuzzy solution can be considered as the optimum designs at different $\alpha-$ the tolerable fortified level. From the viewpoint of cost, the design with the smallest objective of $3854.71 \mathrm{~kg}$

Table 3 Structural Results of Fuzzy Solution

\begin{tabular}{|c|l|l|l|l|l|l|}
\hline$\alpha$ & 1.0 & 0.8 & 0.6 & 0.4 & 0.2 & 0.0 \\
\hline $\begin{array}{c}\mathrm{a}_{\max } \\
(\mathrm{gal})\end{array}$ & 36.99 & 37.79 & 38.57 & 39.25 & 40.18 & 40.11 \\
\hline$\omega_{1}$ & 53.27 & 52.95 & 48.90 & 48.11 & 49.16 & 48.78 \\
\hline$\omega_{2}$ & 83.38 & 83.21 & 80.73 & 79.95 & 80.14 & 80.66 \\
\hline$\omega_{3}$ & 101.55 & 100.19 & 95.30 & 93.88 & 94.31 & 94.77 \\
\hline$\omega_{4}$ & 111.89 & 111.59 & 109.37 & 108.99 & 109.54 & 109.32 \\
\hline$\omega_{5}$ & 133.86 & 134.71 & 133.66 & 133.42 & 134.47 & 134.56 \\
\hline $\begin{array}{c}\sigma_{\max } \\
\left(\mathrm{kg} / \mathrm{mm}^{2}\right)\end{array}$ & 17.46 & 17.20 & 17.97 & 17.62 & 17.78 & 18.08 \\
\hline $\begin{array}{c}\mathrm{obj}^{2} \\
(\mathrm{~kg})\end{array}$ & 5727.41 & 5222.34 & 4583.45 & 4374.27 & 4050.47 & 3854.71 \\
\hline $\begin{array}{c}\mathrm{a}_{\alpha}^{0} \\
(\mathrm{gal})\end{array}$ & 37.00 & 37.80 & 38.60 & 39.40 & 40.20 & 41.00 \\
\hline $\begin{array}{c}\sigma_{\alpha}^{0} \\
\left(\mathrm{kgf} / \mathrm{mm}^{2}\right)\end{array}$ & 18 & 18.02 & 18.04 & 18.06 & 18.08 & 18.10 \\
\hline
\end{tabular}

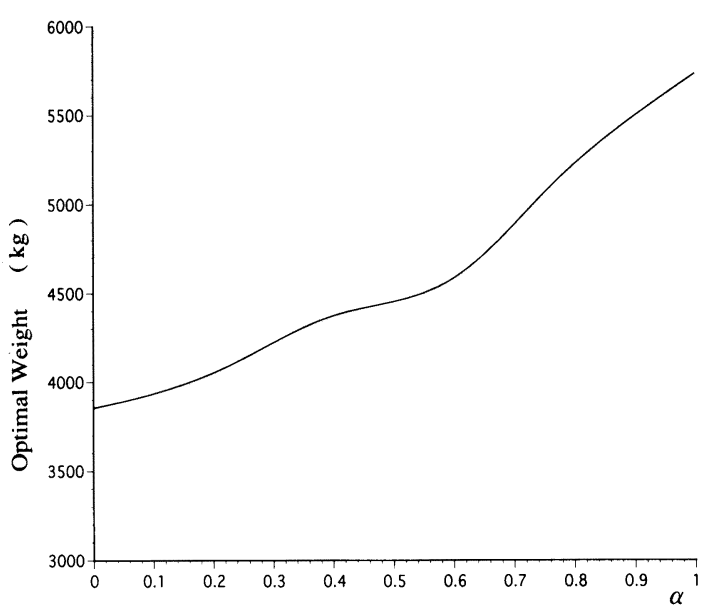

Fig. 6 Fuzzy Solution should be selected. However, from the viewpoint of safety, this solution is the worst choice because the corresponding fortified level $\alpha$ is 0.0 . On the other hand, if the safety of the structure is not sacrificed, the safest structure with $\alpha=1.0$ should be selected, but the corresponding objective of the optimal solution is $5727.41 \mathrm{~kg}$ which is the largest one among all the fuzzy solutions. As stated above, since the fuzziness exists in real engineering problems, especially when the dynamic response is considered, a certain degree of violation of the traditional regulations is tolerable. Therefore, it is not necessary to satisfy the traditional regulations completely to sacrifice the cost of the structure. To select a structure from the fuzzy solutions, designers are in a dilemma. Since the two structures mentioned above can not be selected, a compromise solution should be considered, which should be as safe as possible, and at the same time, whose cost is as small as possible. To achieve this goal, the acceptable values of the objective have to be given by the designers. This means because of the fuzziness, all the costs smaller than a certain value can be considered optimum. Of course, the smaller the objective is, the better the structure is. Therefore, the objective is also described by the degrees of the membership.

In the approach to crisp solution, instead of the minimization of the objective, the membership degree of fuzzy decision is maximized. The admissible region of optimal objective roughly from 3854.71 to 5727.41 obtained from above fuzzy solution is used to establish the membership function of the fuzzy goal. It is desirable that the objective is as small as possible. Therefore, here it is assumed that $\mu_{F}(f)=0$ if the objective $f(\boldsymbol{X}) \geq 5727.41$ and $\mu_{F}(f)=1$ if $f(\boldsymbol{X}) \leq 3854.71$. Table 4 shows the design variables of the optimal design obtained by the approach of crisp solution. The maximal stress, acceleration and the objective value can be found in Table 5. For this crisp solution, the membership degrees of stress constraints, acceleration constraints and the objective are 1.0, 0.6438 and 0.6315 , respectively. The optimum $\lambda(=0.6315)$ is a compromise between the constraints and the objective. From this results, it can be observed that the weight of the crisp solution is smaller than $5727.41 \mathrm{~kg}$ which is obtained in

Table 4 Results of Design Variables in Crisp Solution

\begin{tabular}{|c|c|c|c|c|c|c|c|c|c|c|c|c|}
\hline & \multicolumn{4}{|c|}{ deck } & \multicolumn{4}{c|}{ side } & \multicolumn{4}{c|}{ bottom } \\
\hline$\lambda$ & $\mathrm{h}$ & $\mathrm{t}_{0}$ & $\mathrm{t}_{1}$ & $\mathrm{~b}$ & $\mathrm{~h}$ & $\mathrm{t}_{0}$ & $\mathrm{t}_{1}$ & $\mathrm{~b}$ & $\mathrm{~h}$ & $\mathrm{t}_{0}$ & $\mathrm{t}_{1}$ & $\mathrm{~b}$ \\
\hline 0.6315 & 200.0 & 10.4 & 9.1 & 87.5 & 465.5 & 16.2 & 8.0 & 60.0 & 475.9 & 16.7 & 8.0 & 60.0 \\
\hline
\end{tabular}

Table 5 Structural Results of Crisp Solution

\begin{tabular}{|c|c|c|c|c|c|c|c|c|}
\hline$\lambda$ & $\begin{array}{c}\mathrm{a}_{\max } \\
(\mathrm{gal})\end{array}$ & $\omega_{1}$ & $\omega_{2}$ & $\omega_{3}$ & $\omega_{4}$ & $\omega_{5}$ & $\begin{array}{l}\sigma_{\max } \\
\mathrm{kgf} / \mathrm{mm}^{2}\end{array}$ & $\begin{array}{c}\mathrm{obj} \\
\mathrm{kg}\end{array}$ \\
\hline 0.6315 & 38.42 & 49.40 & 81.74 & 97.21 & 109.30 & 134.10 & 17.85 & 4544.78 \\
\hline
\end{tabular}




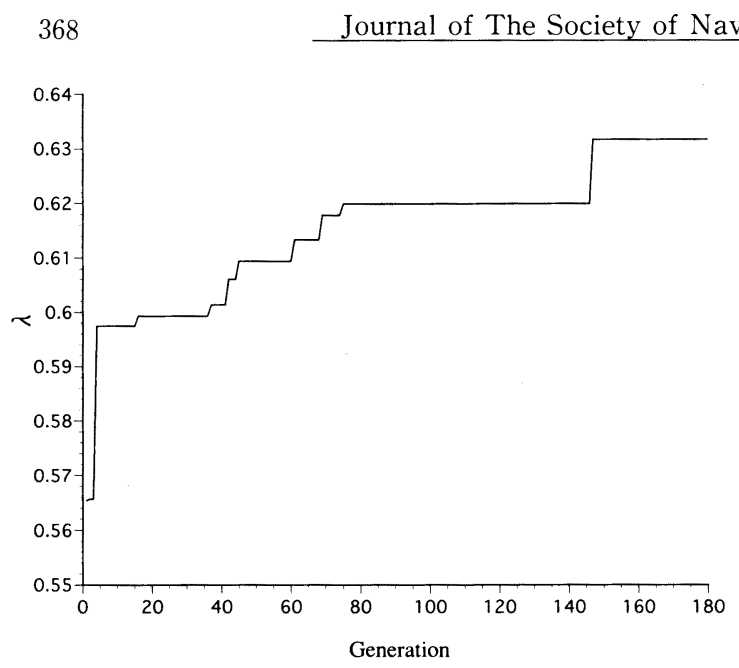

is utilized to handle the constraints. For the crisp solution, the handling of constraints becomes quite simple.

3) The application of the fuzzy theory allows the inclusion of professional wisdom and knowledge into the analytical scheme. However, until the present, this kind of information has not been analyzed completely. In this study, the membership functions are given subjectively. How to obtain them is a problem needed to be studied further.

\section{References}

1) L. Zadeh, Fuzzy Sets, Information and Control, Vol. 8(1965), pp. 338-353.

2) H. J. Zimmerman, Fuzzy Set Theory and Its Application, Second Edition, Kluwer Academic Publishers, Dordrecht, 1991

Fig. 7 History of GA to Crisp Solution

fuzzy solution at $\alpha=1.0$. As known for all, the regulation concerning the acceleration is quite complicated and influenced by many factors. Therefore, the maximal accelerations of structure between $37 \mathrm{gal}$ and $41 \mathrm{gal}$ are acceptable. Obviously the maximal acceleration 38. 42 gal of the crisp solution is also acceptable. Under this tolerable maximal acceleration, the cost of the structure can be saved by the crisp solution. On the other hand, the weight of the crisp solution is larger than $3854.71 \mathrm{~kg}$ which is obtained in fuzzy solution at $\alpha$ $=0.0$. As stated above, the solution with the objective of $3854.71 \mathrm{~kg}$ can not be selected because $\alpha=0.0$ which means that the structure is completely unsafe. However, the membership degree of the constraints for the crisp solution reaches 0.6438 . In other words, the structure in crisp solution is $64 \%$ safe within the acceptable values. The process of the GA to the crisp solution is given in Fig. 7.

\section{Conclusions}

1) The optimization of engine room structure of ship containing fuzzy information in the static and dynamic response constraints and the objective has been presented. Two methods of solving fuzzy optimization problems using GAs are presented along with a numerical example. One method is to calculate the fuzzy solution in which a family of optimal designs under different degrees of satisfaction with the constraints are obtained. The other is to calculate the crisp solution in which the overall degrees of satisfaction with the fuzzy goal and the fuzzy constraints are maximized.

2) The Genetic Algorithm used here seems reliable and convenient for the fuzzy optimization design of ship structures. For the fuzzy solution, the penalty method

3) S. S. Rao, Optimum Design of Structures in a Fuzzy Environment, AIAA Journal, Vol. 25, No. 12 (1987), pp. 1633-1636.

4) Xu Changwen, Xiao Xi, Fuzzy Optimum Design of the Tanker Midship Section with Static and Dynamic Constraints, Int. Shipbuild. Progr., 36, No. 408 (1989), pp. 367-384

5) Tetsuo Okada, Isao Neki, Optimization of Ship Structural Design by Genetic Algorithm, J. Soc. Naval Arch. of Japan, Vol.171 (1992), pp. 259266.

6) Isao Neki, Tetsuo Okada, Optimum Structural Design Using Genetic Algorithm and Finite Element Method, J. Soc. Naval Arch. of Japan, Viol. 177 (1995), pp. 327-337.

7) Zbigniew Michalewicz, Genetic Algorithms+ Data Structures $=$ Evolution Programs, 3rd rev. Springer-Verlag. 1996.

8) Guoqiang Zhou, Hisashi Nobukawa and Fengxiang Yang, Discrete Optimization of Ship Structures from the Viewpoint of Practical Design, J. Soc. Naval Arch. of Japan, Vol. 182 (1997), pp. 551-559.

9) Hisashi Nobukawa, Yang Fengxiang, Mitsuru Kitamura, Zhou Guoqiang, Optimization of Engine Room Structure under Static and Dynamic Constraints Using Genetic Algorithms. J. Soc. Naval Arch. of Japan, Vol. 183 (1998), pp. 315-322.

10) Mitsuru Kitamura, Yang Fengxiang, Hisashi Nobukawa, Design Optimization of Engine Room with Dynamic Response Using Genetic Algorithms. J. Soc. Naval Arch. of Japan, Vol. 186 (1999), pp. 345-353

11) Kaname Taniguchi, Oscillating Pressure near Propellers, Trans. West-Japan Society of Naval Architects, Vol. 16 (1958), pp. 19-30.

12) Study on the tip clearance and axial clearance, Technological Research Report of West-Japan Society of Naval Architects, Vol. 11 (1971).

13) Xianding Jin, Vibration of Ship, Shanghai Jiaotong University. 1988. 